\title{
Phase-contrast X-ray Imaging and Microscopy for Crystallographic Applications at EMBL Beamline P14 of PETRA III
}

\author{
$\underline{\text { Maxim Polikarpov }}{ }^{1, *}$, Gleb Bourenkov ${ }^{1}$, Anatoly Snigirev $^{2}$ and Thomas Schneider ${ }^{1}$ \\ 1. European Molecular Biology Laboratory (EMBL), Hamburg Unit c/o DESY, Notkestrasse 85, 22603 \\ Hamburg, Germany. \\ 2. Immanuel Kant Baltic Federal University, Nevskogo 14, 236041 Kaliningrad, Russia. \\ * Corresponding author, mpolikarpov@embl-hamburg.de
}

EMBL Hamburg operates the P14 beamline at the high-brilliance PETRA III storage ring at DESY (Hamburg, Germany). Delivering hard X-rays with energies of $6-30 \mathrm{keV}, \mathrm{P} 14$ is very versatile: shape, size and intensity of the X-ray beam are easily tunable by using both reflective and refractive optical elements. Sample stage and detecting systems are state-of-the-art including a high-precision vertical goniometer (developed in collaboration with EMBL Grenoble and ARINAX (Voiron, France), a robotized sample changer (developed in-house) and a DECTRIS (Baden, Switzerland) EIGER 16M detector. These features enable crystallography of large macromolecular complexes, serial crystallography, time-resolved and in-situ diffraction data collection.

Of special interest in macromolecular crystallography (MX) are crystals grown in lipidic cubic phase (LCP) which - when cooled - is opaque for visible light (fig. 1, a). To make visualization of LCPcrystals feasible, a diffraction raster-scans are implemented on many MX beamlines [1-2]. These are based on rastering of the sample with a micron-scale X-ray beam while recording a diffraction signal from each area. The beam size (typically 5-10 $\mu \mathrm{m}$ ) limits the raster-scan resolution while crystals often need to be located with a sub-micron accuracy. Exploiting the flexibility of the beamline optics, we are investigating new methods and techniques to look into samples of interest via full-field phase-contrast X-ray imaging [3-4] and microscopy. Unlike conventional absorption X-ray imaging, phase contrast renders interfaces and density gradients clearly visible in a biological sample. Thus, we applied phasecontrast X-ray imaging for full-field visualization of lysozyme crystals in LCP with submicron resolution (fig.1, b, c). The sample was exposed to $15 \mathrm{keV}$ X-rays. Images were recorded in the edgeenhancing phase-contrast regime at $11 \mathrm{~cm}$ downstream the sample with a high-resolution X-ray CCD camera (pixel size of $0.6 \mu \mathrm{m}$ ). A $20 \mathrm{msec}$-exposure was sufficient to visualize crystals at submicron resolution limited only by the pixel size of the camera. Besides the high resolution, X-ray imaging delivered almost negligible radiation dose of $\sim 100 \mathrm{~Gy}$ which is a neglible fraction of the tolerable dose for a typical cryo-cooled crystal (20 MGy). Imaging thus provides clear advantages in comparison with conventional raster-scan technique which delivers a dose of $\sim 1 \mathrm{MGy}$ during an exploratory scan.

To achieve higher resolution and to see fine details inside a crystal of interest, we also implement X-ray refractive lenses as an objective in the microscopy mode. This approach allows us to magnify details of the sample up to 15 times prior to the optical detection of X-rays that carry the phase-contrast information from the interaction with sample. With the current setup, a sub-100nm resolution can be achieved and it is possible to visualize crystal boundaries and local deformations in situ. High-precision rotation of the sample stage at P14 also allows to perform X-ray tomography. Due to the use of highenergy X-rays for the imaging process, materials and tissues with thicknesses in the mm-range can be penetrated. The latter also allows P14 to look forward for in-vivo and time-resolved experimental applications with other biological objects. 


\section{References:}

[1] V. Cherezov et al, Journal of The Royal Society Interface 6 (2009), S587-S597.

[2] M. Bowler et al, Acta Crystallographica Section D 66 (2010), 855-864.

[3] A. Snigirev et al, Review of Scientific Instruments 66 (1995), 5486.

[4] S. Brockhauser et al, Journal of Applied Crystallography 41 (2008), 1057-1066.
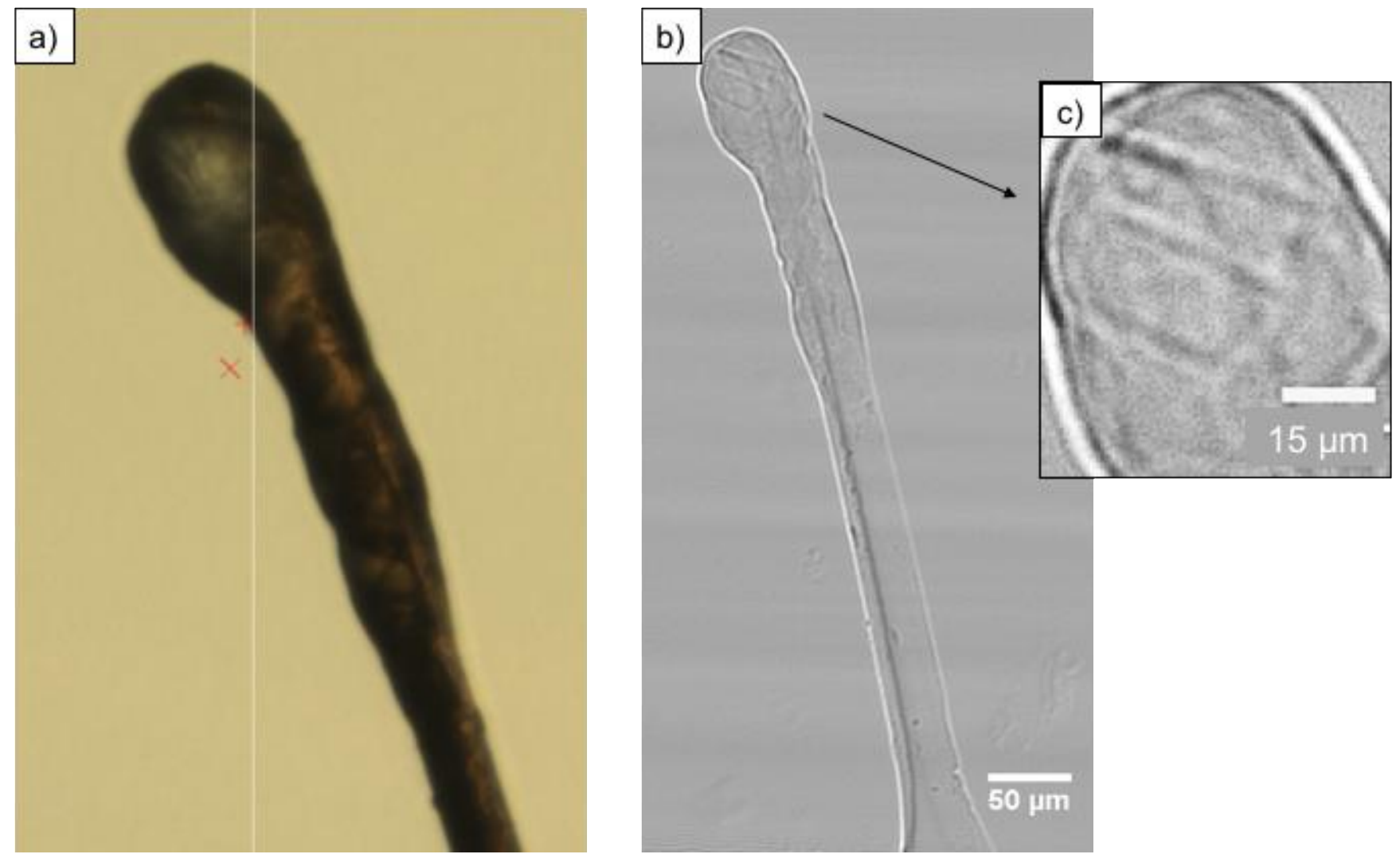

Figure 1. LCP containing crystals is opaque to visible light (a). X-ray phase-contrast imaging (b, c) clearly visualizes crystals in the LCP-matrix. 